\title{
Comprensión de los Conceptos de Campo, Energía y Potencial Eléctricos y Magnéticos en Estudiantes Universitarios
}

Comprehension of electric and magnetic field, energy and potential concepts in undergraduate students

\author{
Sandra Velazco, Julia Salinas \\ Facultad de Ciencias Exactas y Tecnología, Universidad Nacional de Tucumán \\ Av. Independencia 1800, (4000) Tucumán, Argentina \\ E-mail: svelazco@herrera.unt.edu.ar; jsalinas@herrera.unt.edu.ar
}

Recebido em 21 de Novembro 2000. Manuscrito revisado em 01 de Julho 2001. Aceito em 06 de Agosto 2001.

\begin{abstract}
La enseñanza y el aprendizaje de los conceptos de campo, potencial y energía plantea especiales dificultades a estudiantes y docentes. Informamos sobre un estudio en el área de la electricidad y el magnetismo. Se analiza la comprensión de aspectos básicos de aquellos conceptos alcanzada por alumnos de segundo año de carreras de Ingeniería que habían completado el cursado de la asignatura y aprobado las evaluaciones correspondientes. Los resultados muestran la necesidad de complementar más la instrucción con abordajes cualitativos, conceptuales, comprensivos, que en la instrucción habitual suelen ser postergados por tratamientos casi excluyentemente centrados en formalismos matemáticos cuyo significado físico no queda claro ante los estudiantes.
\end{abstract}

\begin{abstract}
Learning and teaching field, energy and potential concepts electromagnetism present special difficulties to students and teachers. We inform on a study in domain. Comprehension of basic aspects related with those concepts attained by engineering students who had completed the course of electricity and magnetism is analysed. Results show the necessity of complementary qualitative and quantitative analysis in instruction. In traditional instruction qualitative treatments are usually delayed by treatments almost entirely centred in mathematics formalisms, whose physical mean is not clear for students.
\end{abstract}

\section{Introducción y Planteamiento del Problema}

La formulación axiomática del electromagnetismo clásico muestra que hay un número limitado de conceptos, muy potentes, que intervienen en la explicación y predicción de una amplia variedad de fenómenos. Las nociones de campo, potencial y energía se encuentran entre esas entidades conceptuales.

La introducción en la física de modelos explicativos basados en la idea de campo supuso históricamente un avance significativo con respecto a las teorías de acción instantánea a distancia y permitió explicar nuevos efectos.

Mientras las teorías de acción a distancia suponen que la interacción se ejerce en forma instantánea, sin que se brinde ninguna explicación procedimental sobre el modo en que un cuerpo produce efectos sobre otro, las teorías de campo introducen mediadores para la transferencia de la interacción: las ideas de campo y potencial.
Se supone, por ejemplo, que la presencia de un cuerpo cargado "distorsionará" o "creará una condición en el espacio" tal que, si introducimos otro cuerpo cargado, éste experimentará una fuerza. Tal "potencialidad" para producir una fuerza se denomina campo eléctrico (Feynman, Leighton, Sands 1972).

El nuevo modelo supone también que los campos y potenciales persistirán aún en ausencia de cargas testigo que los pongan en evidencia.

En la explicación de los fenómenos electrostáticos y magnetostáticos, la teoría de acción a distancia y la teoría de campo pueden considerarse como dos modelos conceptuales o intrepretaciones alternativas, potencialmente útiles para estudiar aquellos comportamientos (Furió y Guisasola 1997).

Sin embargo, otros efectos no pueden comprenderse en el marco de la acción instantánea a distancia: si una carga se mueve de manera arbitraria, se encuentra que la acción sobre otra carga no cambia instantáneamente, sino que sufre un retardo. La teoría de campo da cuenta de estos fenómenos. La solución general de las ecuacio- 
nes de Maxwell en situaciones dependientes del tiempo predice que los efectos de una carga o corriente sobre un punto se propagarán desde la fuente a velocidad finita, más precisamente a la velocidad de la luz (Feynman, Leighton, Sands 1972). Esta predicción es coherente con la idea de que las perturbaciones se transmiten paso a paso, desde la fuente y a través de cada parte del campo sobre la contigua, y apoya el significado del campo electromagnético como el agente transmisor de las acciones.

Por otro lado, el modelo de campo posibilitó la unificación, en un único marco conceptual, de la electricidad, el magnetismo y la óptica, que surgieron históricamente como dominios inconexos dentro de la física.

La potencia y fertilidad de la teoría electromagnética clásica va sin embargo acompañada de una elevada complejidad. Sus nociones fundamentales, como la idea de campo, presentan un alto nivel de abstracción, que se acentúa para conceptos aún más elaborados e integradores como los de potencial o energía.

Las investigaciones educativas en electromagnetismo son aún incipientes, comparadas con las que se desarrollan en otras áreas de la física como mecánica, termodinámica u óptica.

La problemática planteada por la enseñanza y el aprendizaje del comportamiento de circuitos eléctricos ha sido probablemente la más investigada en este campo (por ejemplo Cohen, Eylon y Ganiel 1983; Danusso y Dupré 1987; Heller 1987; Kärrqvist 1987; Varela y otros 1988; Dupin y Joshua 1990; Millar y Lim Beh 1993). Algunos autores han propuesto estrategias superadoras para la secuenciación de la enseñanza del electromagnetismo (Cudmani y Fontdevila 1990; Meneses y Caballero 1995). Pero es relativamente poco lo que se ha avanzado en relación con la comprensión que alcanzan los estudiantes sobre conceptuaciones fundamentales de la teoría electromagnética clásica (Solbes y Quero 1991; Viennot y Rainson 1992; Furió y Guisasola 1993, Törnkvist y otros 1993; Guisasola y Furió 1994; Rainson, Tranströmer y Viennot 1994; Galili 1995).

En este trabajo se presentan resultados obtenidos en una tesis de Licenciatura en Física (Velazco 1998). Aquí se realiza un control experimental de la comprensión alcanzada por estudiantes universitarios sobre nociones básicas del electromagnetismo clásico (el concepto de campo, de potencial electrostático y de energía) al término de la instrucción; se sugieren posibles razones para las incomprensiones detectadas y se presentan algunos criterios potencialmente útiles para enriquecer la enseñanza y propiciar aprendizajes más significativos.

\section{Hipótesis de Trabajo y Diseño Experimental}

A partir de la experiencia docente y de la consulta de bibliografía sobre investigaciones educativas en electromagnetismo formulamos las siguientes hipótesis de trabajo referidas al aprendizaje de los conceptos de campo, potencial y energía:

$\mathbf{H}_{1}$ ) Muchos alumnos utilizan modelos no científicos para el campo.

$\mathbf{H}_{2}$ ) Muchos alumnos tienen dificultad para comprender la relevancia del concepto de potencial eléctrico o sus propiedades.

$\mathbf{H}_{3}$ ) Muchos alumnos establecen una dependencia incorrecta entre el potencial eléctrico en un punto del espacio y una carga de prueba ubicada en ese punto.

$\mathbf{H}_{4}$ ) Muchos alumnos no comprenden la dependencia del valor del potencial eléctrico o de la energía potencial eléctrica con el referencial elegido.

Para controlar estas hipótesis se elaboraron 16 enunciados de situaciones problemáticas cualitativas, sencillas pero conceptualmente relevantes, que fueron administradas a 115 estudiantes de segundo año de carreras de Ingeniería de la Universidad Nacional de Tucumán. Todos los estudiantes de la muestra habían completado el cursado de la asignatura (clases teóricas, sesiones teórico - prácticas de resolución de problemas y realización de experiencias de laboratorio) y habían aprobado las evaluaciones correspondientes.

Los enunciados debían responderse por escrito, individualmente, y podían consultarse libremente libros de textos de uso habitual en ciclos básicos universitarios (por ejemplo Halliday et all, Tipler) durante la resolución.

Para la elaboración de los cuestionarios se realizó una experiencia piloto que permitió una posterior selección y / o modificación de los enunciados, algunos de los cuales están basados o reproducen situaciones problemáticas utilizadas por otros autores.

En el Apéndice se presentan los enunciados propuestos y breves comentarios sobre respuestas científicamente correctas a los mismos.

\section{Presentación y análisis de los resultados}

\section{Resultados obtenidos al contrastar $\mathbf{H}_{1}$}

Recordemos que la hipótesis $\mathbf{H}_{\mathbf{1}}$ afirma que $\mathbf{M u -}$ chos alumnos utilizan modelos no científicos para el campo. (El número de estudiantes interrogados en este caso fue de 86 , distribuidos entre los problemas $\mathrm{N}^{\mathrm{O}} \mathbf{1}, \mathbf{2}, \mathbf{3}, \mathbf{4}, \mathbf{5}, \mathbf{6}$ y $\left.\mathbf{7}\right)$.

$\mathrm{El}$ análisis de las respuestas permitió identificar las siguientes categorías: 


$$
\mathbf{N}=86
$$

\begin{tabular}{|l|c|c|c|}
\hline \multirow{2}{*}{$\begin{array}{l}\text { Respuestas } \\
\text { Incorrectas }\end{array}$} & $\begin{array}{c}\text { fundadas en un modelo no científico } \\
\text { de campo explicitado por el estudiante }\end{array}$ & 30 & $35 \%$ \\
\cline { 2 - 4 } & sin explicitación de un modelo de campo & 23 & $27 \%$ \\
\hline Respuesta confusa - No responde & & 19 & $22 \%$ \\
\hline Respuesta correcta & & 14 & $16 \%$ \\
\hline
\end{tabular}

"Respuestas incorrectas fundadas en un modelo no científico de campo explicitado por el estudiante"

Los estudiantes incluidos en esta categoría, un $35 \%$ de la muestra, parecen manifestar en sus respuestas un modelo material para el campo o las líneas de campo.

En los problemas $\mathbf{1}$ y $\mathbf{2}$ (en los que se pide analizar el campo de un imán o de un dipolo y no el campo resultante) algunos alumnos dibujan líneas de campo que se desvían o deforman en presencia de una placa de madera o que se interrumpen en la región ocupada por aquélla. Otros estudiantes parecen suponer que la lámina "apantalla" la influencia de una de las cargas o de un trozo de imán en la región ocupada por la otra carga o la otra parte del imán y dibujan líneas de campo de sólo una carga o un imán en cada región.

La idea de que el campo eléctrico o magnético de un determinado sistema de cargas o imanes se modifica por la presencia de un obstáculo material, sin que se discrimine entre el campo producido por ese sistema y el campo resultante, también aparece en los problemas 3, 4, 5 y 6. En ellos, los estudiantes parecen considerar al campo como alguna entidad que se propaga desde la fuente, alterándose al encontrar obstáculos físicos interpuestos en su camino.

Así, alumnos que responden a los problemas 3 y 4 (en los que se pide analizar el campo de un imán o de una carga y no el campo resultante) consideran que una placa de madera es una barrera "permeable" al campo y hacen afirmaciones del tipo: "La madera deja pasar el campo eléctrico". Otros atribuyen a la lámina un carácter "impermeable" y hacen referencia a su "naturaleza aisladora". Reproducimos una respuesta típica: "Debido a que la placa de madera actúa como aislante y es infinita, el campo generado por Q no puede atravesarla". Ninguno de estos estudiantes menciona efectos de polarización o magnetización de la placa.

En los problemas 5 y 6 (en los que se pide analizar el campo de una carga y no el campo resultante) otros alumnos sostienen que una barrera material, en este caso un conductor, evitará que una carga actúe en forma directa en un punto $\mathrm{P}$ ubicado detrás de esa barrera. Parecen considerar que el campo de la carga Q se extenderá solamente en la región limitada por el conductor, donde inducirá cargas que a su vez serán las únicas fuentes del campo que actúa en P. Así, encontramos respuestas del tipo: "Sí. Se induce carga en la superficie del conductor y ésta crea un E en P" (prob. $5)$.

\section{"Respuestas incorrectas sin explicitación de un modelo de campo"}

Los alumnos incluidos en esta categoría no manifiestan explícitamente a través de sus respuestas un modelo interpretativo para el campo, pero le atribuyen comportamientos diferentes de los que se les asignaría en un contexto científico.

Muchas respuestas de esta categoría (al igual que otras correspondientes a la categoría anterior) muestran que los aprendices tienen dificultades para aplicar correctamente el principio de superposición: parecen no discriminar un campo resultante de sus contribuciones parciales. Así, por ejemplo, en el problema 5 (en el que se interroga sobre el campo de una carga y no sobre el campo resultante) afirman que "el campo de $Q$ en $\mathrm{P}$ es cero porque el campo eléctrico en un conductor siempre es cero" (aunque esto sea válido sólo para el campo eléctrico resultante en un conductor en equilibrio estático). En los problemas 3 y 6, admiten que la carga $Q$ crea un campo eléctrico en $P$, pero lo calculan usando el teorema de Gauss, a pesar de que esta ley involucra el campo eléctrico resultante en cada punto de la superficie gaussiana; por otra parte, no advierten que en estas situaciones no se cumplen las condiciones de simetría necesarias para aplicar aquella ecuación al cálculo del campo total.

Finalmente, otros alumnos que responden al problema 3 afirman que es necesaria una carga en $\mathrm{P}$ para que haya campo en ese punto. En el ítem c) del problema 7 no dibujan nada cuando no hay carga en $P$. Estos estudiantes parecen necesitar una carga testigo para aceptar la presencia del campo en un punto.

\section{Resultados obtenidos al contrastar $\mathbf{H}_{2}$}

Recordemos que la hipótesis $\mathbf{H}_{\mathbf{2}}$ afirma que $\mathbf{M u}$ chos alumnos tienen dificultad para comprender la relevancia del concepto de potencial eléctrico o sus propiedades. (Para esta contrastación experimental se interrogaron 56 estudiantes distribuidos de la siguiente forma: 11 alumnos que respondieron al problema 8, 18 que respondieron al problema 9, 14 al problema $\mathbf{1 0}$ y 13 al problema 11).

El análisis de las respuestas permitió identificar en este caso las siguientes categorías: 
$\mathbf{N}=56$

\begin{tabular}{|l|c|c|}
\hline Incomprensión de la relevancia de V o sus propriedades & 34 & $61 \%$ \\
\hline Otras respuestas & 5 & $9 \%$ \\
\hline Respuesta confusa / no responde / no sabe / no justifica & 14 & $25 \%$ \\
\hline Respuestas incorrectas que utilizan el concepto de potencial & 3 & $5 \%$ \\
\hline
\end{tabular}

\section{"Incomprensión de la relevancia de V o sus pro- piedades"}

En esta categoría cae más de la mitad de las respuestas de los estudiantes.

Ubicamos aquí respuestas dadas al problema 8, en las que los estudiantes manifiestan que circulará carga positiva del cascarón a la tierra o que habrá un flujo de carga negativa de la tierra al cascarón, hasta que aquél se neutralice. Ninguno menciona el concepto de potencial eléctrico, magnitud que se utiliza en una explicación científicamente correcta para explicar el flujo de carga y para especificar la condición de equilibrio estático en un sistema de conductores.

Estos resultados sugieren que los alumnos no comprenden la relevancia del concepto de potencial eléctrico para explicar el comportamiento del sistema bajo estudio, suposición reforzada por respuestas obtenidas en los problemas $\mathbf{9}, \mathbf{1 0}$ y $\mathbf{1 1}$ :

En el problema $\mathbf{9}$ los aprendices no utilizan la idea de que, en equilibrio estático, el potencial debe ser el mismo en el conductor y en la tierra. Se considera incorrectamente que la carga neta del cuerpo conectado a tierra determinará su potencial y no se lo relaciona con el potencial de la tierra.

En el problema 10, ninguno de los alumnos cuyas explicaciones se encuadran en esta categoría menciona el concepto de potencial. La mayoría responde que no circulará carga; admiten, aparentemente, que el sistema está en equilibrio cuando las cargas de ambas esferas son iguales. Los otros afirman que habrá circulación hasta que se igualen las densidades superficiales de carga de ambos cuerpos.

Finalmente, en el problema 11, muchos estudiantes ignoran la información referida al potencial eléctrico (a pesar de que es la única relevante) y consideran que las propiedades del medio determinarán unívocamente el campo eléctrico en cada zona: afirman enfáticamente que "el campo eléctrico es cero en el conductor" (probablemente extrapolan incorrectamente un resultado memorizado en electrostática) o comparan la intensidad del campo eléctrico en las regiones I y III a través de una dependencia excluyente con la constante dieléctrica $\mathrm{K}$ como la única condición significativa.

\section{"Otras respuestas"}

Incluimos aquí respuestas al problema 9 donde se afirma que el potencial de la tierra es cero por convención y que el potencial del conductor es cero por estar conectado a tierra, sin brindar ninguna explicación adicional que permita conocer la forma en que se razona.

En otras respuestas los estudiantes invocan el concepto de potencial pero parecen combinarlo con sus ideas intuitivas sobre la causa del movimiento de cargas y la condición de equilibrio estático en los conductores: en el problema 9 explican que habrá un flujo de carga hasta que las cargas del cascarón y la tierra se igualen, afirmando que en esta situación los potenciales de ambos cuerpos serán los mismos y el sistema estará en equilibrio, o en el problema $\mathbf{1 0}$ afirman que la igualdad de potenciales entre los conductores equivale a la igualdad de cargas o de densidades superficiales de carga.

\section{Resultados obtenidos al contrastar $\mathbf{H}_{3}$}

Recordemos que la hipótesis $\mathbf{H}_{\mathbf{3}}$ afirma que $\mathbf{M u -}$ chos alumnos establecen una dependencia incorrecta entre el potencial eléctrico en un punto del espacio y una carga de prueba ubicada en ese punto. (Para esta contrastación se interrogó un total de 30 alumnos: 14 que respondieron al problema 12 y 16 al problema 13).

Las respuestas se organizaron en las siguientes categorías:$$
\mathbf{N}=\mathbf{3 0}
$$

\begin{tabular}{|l|c|c|}
\hline Dependencia incorreta entre $V$ y $q_{\text {prueba }}$ & 17 & $57 \%$ \\
\hline Respuesta confusa / no sabe / no justifica & 8 & $27 \%$ \\
\hline Respuesta correcta & 5 & $16 \%$ \\
\hline
\end{tabular}

\section{"Dependencia incorrecta entre V y q prueba"}

Más de la mitad de los estudiantes que responden a estos problemas consideran incorrectamente que el po- tencial eléctrico en un punto depende de la carga de prueba ubicada en ese punto.

Algunos interpretan la fórmula "V $=\mathrm{k} \mathrm{q} / \mathrm{r}$ ", suponiendo que "q" es la carga ubicada en el punto donde se 
calcula V. Otros alumnos afirman, sin mencionar ninguna ecuación, que el potencial es proporcional a la carga localizada en el punto considerado.

Otros estudiantes explican el comportamiento del potencial en un punto atribuyéndole propiedades semejantes a las que asignan al campo eléctrico en ese mismo punto. El campo, a su vez, dependería de la carga de prueba. De este modo, brindan respuestas del tipo "El potencial depende del campo y éste a su vez de la carga q" (problema 13).

\section{Resultados obtenidos al contrastar $\mathbf{H}_{4}$}

Recordemos que la hipótesis $\mathbf{H}_{\mathbf{4}}$ afirma que $\mathbf{M u -}$ chos alumnos no comprenden la dependencia del valor del potencial eléctrico o de la energía potencial eléctrica con el referencial elegido. (Para esta contrastación se interrogaron 48 alumnos: 12 que respondieron al problema 14, 16 al problema 15 y 20 problema 16).

Las categorías identificadas fueron las siguientes:

$$
\mathbf{N}=48
$$

\begin{tabular}{|l|c|c|}
\hline No mencionan una dependencia con el referencial & 24 & $50 \%$ \\
\hline Mencionan una dependencia con el referencial & 8 & $17 \%$ \\
\hline No justifica / no sabe / no responde / respuesta confusa & 16 & $33 \%$ \\
\hline
\end{tabular}

\section{"No mencionan una dependencia con el referen- cial"}

La elaboración de una respuesta científicamente correcta a los problemas planteados en la contrastación de $\mathbf{H}_{4}$ requería tener en cuenta, entre otros factores, que el valor numérico del potencial eléctrico o la energía potencial eléctrica dependen del referencial elegido para especificarlos. No obstante, la mitad de los estudiantes interrogados no mencionan ninguna dependencia entre aquellas magnitudes y el referencial.

Muchos parecen basar su respuesta en un razonamiento que no considera las condiciones de validez de las ecuaciones, en particular la adopción implícita de un referencial al utilizar una fórmula dada para el potencial o la energía potencial eléctrica. Estos alumnos escriben y comentan ecuaciones matemáticas para el potencial eléctrico (en el problema 14) o para la energía potencial eléctrica de una carga puntual (en los problemas 15 y 16), sin mencionar el referencial. Por ejemplo: "V = k q / r es mayor que cero si q es mayor que cero" (problema 15).

Otros estudiantes, aunque no escriben ecuaciones parecen basar su respuesta en la interpretación, también puramente algorítmica, de alguna fórmula. Por ejemplo, en el problema 14 uno de ellos explica: "el signo de V depende sólo del signo de q, pues las distancias siempre son positivas".

Otros alumnos consideran que la energía potencial de una carga puntual depende del campo eléctrico en la posición de esa carga. Al responder al problema $\mathbf{1 5}$ explican que " $U_{\text {pot }}$ depende de la carga y del campo que actúa sobre ella".

\section{Comentarios finales}

Las hipótesis parecen verificarse ampliamente a partir de la evidencia empírica obtenida: los estudiantes no utilizan en sus respuestas interpretaciones cien- tíficamente correctas para los conceptos de campo, potencial eléctrico y energía eléctrica.

En relación a $\mathbf{H}_{1}$, los aprendices atribuyen propiedades no científicas a la idea de campo:

- Porcentajes significativos de alumnos utilizan o brindan respuestas coherentes con una interpretación del concepto de campo que difiere de modelos científicos válidos dentro del electromagnetismo clásico.

Así, muchos estudiantes asignan al campo un comportamiento material. Suponen que las líneas de campo serán interrumpidas o desviadas por una placa de madera o bien que la lámina apantallará la influencia de una carga o un trozo de imán en la región posterior a la placa. El hecho de dibujar líneas de campo parece confundir a muchos alumnos (Törnskvist y otros 1991) que tratan estas representaciones gráficas abstractas como curvas materiales o lineas de flujo que pueden ser distorsionadas en presencia de un límite físico.

Otros estudiantes describen la acción de una carga o imán sobre un punto mediante una explicación secuencial incorrecta en la que el campo, concebido como una única entidad, se iría modificando al encontrar obstáculos físicos interpuestos en su camino. Este tipo de explicación basada en una secuencia de eventos simples, donde cada evento se considera la causa del siguiente ha sido denominada "razonamiento causal lineal" y ha sido detectada también por otros autores en la interpretación del concepto de campo eléctrico (Viennot y Rainson 1992).

- Una gran proporción de alumnos no aplica el principio de superposición de campos. Éste resulta incompatible con las explicaciones secuenciales citadas y con otras que manifiestan una no diferenciación, por parte de los estudiantes, entre un campo resultante y sus contribuciones parciales.

- Algunos alumnos precisan una carga de prueba en un punto para aceptar la presencia del campo en ese 
punto, manifestando dificultades para concebir a esa entidad como una propiedad del espacio vacío.

Los resultados obtenidos al contrastar $\mathrm{H}_{2}$, $\mathbf{H}_{3}$ y $\mathbf{H}_{4}$ muestran que los estudiantes manifiestan especiales dificultades para comprender el concepto de potencial electrostático y de energía potencial eléctrica.

- Muchos alumnos no advierten la relevancia de la idea de potencial: en situaciones donde resulta imprescindible utilizar aquella conceptuación física para explicar correctamente el comportamiento del sistema bajo estudio, no mencionan la noción de potencial eléctrico, ignoran información relevante sobre esa magnitud, o no emplean sus propiedades.

En particular, no utilizan la idea de potencial para explicar el flujo de carga y el equilibrio estático en cuerpos conductores y usan solamente la noción de carga o densidad superficial de carga para dar cuenta de esos fenómenos, resultados que convergen con los de otras investigaciones donde se advierte que, en un circuito, a la diferencia de carga o densidad volumétrica de carga se atribuye la causa de la corriente (Benseghir y Closset 1996). Para los estudiantes sería la "cantidad de carga" o "densidad superficial de carga" las magnitudes que regularían el movimiento de carga y que especificarían la condición de equilibrio estático en un sistema de conductores. Estas respuestas podrían reflejar un cierto "modelo de fluido" para la carga, propuesto también por otros autores (Furió y Guisasola 1993; Guisasola y Furió 1994).

Cuando se brinda información pertinente sobre el potencial y se solicitan predicciones sobre el comportamiento del campo eléctrico, los alumnos no razonan en términos de $\mathrm{V}$ y asignan a $\mathrm{E}$ una dependencia excluyente con las propiedades del medio material.

- Porcentajes importantes de estudiantes vinculan incorrectamente el potencial eléctrico en un punto a la carga de prueba ubicada en ese punto. Así, muchos alumnos suponen que el potencial será proporcional a la magnitud de la carga testigo, que cambiará de signo al modificar el signo de la carga de prueba o que no persistirá en ausencia de aquella carga. Este tipo de respuesta, similar a la obtenida cuando se interroga sobre el campo, podría manifestar dificultades, por parte de los estudiantes, para independizar nociones abstractas de algún soporte físico.

- Un número significativo de estudiantes asigna al potencial eléctrico en un punto un único valor, sin considerar en sus respuestas la adopción implícita de un determinado referencial. Cuando son interrogados sobre la energía potencial eléctrica de una carga puntual manifiestan dificultades similares. Estos resultados podrían atribuirse a un uso puramente mecánico de fórmulas matemáticas o bien a la tendencia de algunos estudiantes a atribuir un carácter "absoluto" a ciertas magnitudes.

La revisión de antecedentes sobre el tema muestra que aquella tendencia ha sido registrada por otros autores (por ejemplo Villani y Pacca 1987 y 1990) en diferentes dominios de la física tales como mecánica y relatividad.

- También hemos detectado, en un pequeño grupo de respuestas, que al ser interrogados sobre el potencial eléctrico en un punto, los estudiantes le confieren el comportamiento de otras magnitudes físicas, tales como la carga ubicada en esa posición o el campo eléctrico asociado a ese punto del espacio. Cuando responden sobre la energía potencial eléctrica de una carga puntual, atribuyen a la energía las propiedades del campo en la posición de la carga.

En síntesis, a posteriori de la instrucción muchos estudiantes universitarios muestran serias limitaciones en la comprensión de conceptos tan fundamentales como los de campo, potencial y energía. Muchos aprendices, que pueden mostrase hábiles para resolver problemas aplicando el formalismo matemático que utiliza la física, no acompañan ese abordaje de una interpretación científicamente correcta de los elementos conceptuales involucrados. Por ello, parece necesario que los docentes seamos conscientes de las dificultades que plantea el tema y destinemos en la enseñanza un tiempo suficiente a actividades tendientes a que los estudiantes comprendan las características básicas, los aspectos distintivos e importantes alcances de estas poderosas herramientas conceptuales. Cabe destacar la relevancia en la instrucción de discusiones cualitativas (además de las cuantitativas) que favorezcan y profundicen una comprensión más significativa del cuerpo de conocimiento de la física.

\section{Referencias bibliográficas}

- Benseghir, A. y Closset, J.L. 1996, The electrostatics - electrokinetics transition: historical and educational dificulties, International Journal of Science Education, 18 (2), 179-191.

- Cohen R., Eylon B., Ganiel V., 1983, Potential difference and current in simple electric circuits: a study of students' concepts, American Journal of Physics, 51 (5), 407-412.

- Cudmani L. C. de, Fontdevila P., 1990, Concepciones previas en el aprendizaje significativo del electromagnetismo, Enseñanza de las Ciencias, 8 (3), 215-222.

- Danusso L., Dupré F., 1987, Student representations of simple circuits, Proceedings of the Second International Seminar "Misconceptions and Educational Strategies in Sciences and Mathematics" (Cornell university, Ithaca, New York, July 1987).

- Duphin J., Joshua, 1990, Una analogía térmica para la enseñanza de la corriente continua en electricidad: descripción y evaluación, Enseñanza de las Ciencias, 8 (2), 119-126.

- Feynman R., Leighton R., Sands M., 1972, Vol I: Mecánica, Radiación y Calor, Vol. II: Electromag- 
netismo y materia (Fondo Educativo Interamericano, EEUU).

- Furió, C. y Guisasola, J. 1993, ¿Puede ayudar la historia de la ciencia a entender por qué los estudiantes no comprenden los conceptos de carga y potencial eléctrico?, Revista Española de Física, 7 (3), 46-50.

- Furió C., Guisasola J.,1997, Deficiencias epistemológicas en la enseñanza habitual de los conceptos de campo y potencial eléctricos, Enseñanza de las Ciencias, 15 (2), 259-271.

- Galili, I. 1995. Mechanics background influences students conceptions in electromagnetism, International Journal of Science Education, 17 (3), 371-387.

- Guisasola J., Furió C., 1994, Dificultades en el aprendizaje significativo de algunos conceptos de electrostática, Investigación en la escuela, 23, 103-114.

- Halliday D., Resnick R., Krane K., 1998, Física, Vol. II (Compañía Editorial Continental, México).

- Heller P., 1987, Use of core propositions in solving current electricity problems, Proceedings of the Second International Seminar "Misconceptions and Educational Strategies in Sciences and Mathematics" (Cornell university, Ithaca, New York, July 1987).

- Jackson J. D., 1966, Electrodinámica Clásica (Editorial Alhambra, Madrid).

- Kärrqvist C., 1987, Pupils are able, Proceedings of the Second International Seminar "Misconceptions and Educational Strategies in Sciences and Mathematics" (Cornell university, Ithaca, New York, July 1987).

- Meneses, J. Y Caballero, M. 1995, Secuencia de enseñanza sobre el electromagnetismo, Enseñanza de las Ciencias, 13 (1), 36-45.

- Millar R., Lim Beh K., 1993, Students' understanding of voltage in simple parallel electric circuits, International Journal of science Education, 15 (4), 351-361.

- Rainson S., Tränstromer G., Viennot L., 1994, Students' understanding of superposition of electric fields, American Journal of Physics, 62 (11), 1.026-1.032.

- Solbes, J. Y Quero, J. 1991, Análisis de la Introducción del concepto de campo, Revista Española de Física, 5 (3), 34-39.

- Tipler P., 1996, Física, Vol. II (Editorial Reverté, Barcelona).

- Törnkvist, S.; Petterson, A. y Tränstromer, G. 1993, Confusion by representation: on students' comprehension on the electric field concept, American Journal of Physics, 61 (4), 335-338.

- Varela P., Manrique M., Favieres A., 1988, Circuitos eléctricos: una aplicación de un modelo de enseñanza aprendizaje basado en las ideas previas de los alumnos, Enseñanza de las Ciencias, 6 (3), 285-290.

- Velazco, S. 1998. El campo electromagnético en la enseñanza y el aprendizaje de la física, Trabajo Final de Licenciatura en Física (Instituto de Física, FaCEyT, UNT).

- Viennot, L. y Rainson, S. 1992, Students reasoning about the superposition of electric fields, International
Journal of Science Education, 14 (4), 475-487.

- Villani, A. y Pacca, J. 1987, Students' spontaneus ideas about the speed of light, International Journal of Science Education, 9 (1), 55-66.

- Villani, A. y Pacca, J. 1990, Spontaneus reasoning of graduate students, International Journal of Science Education, 12 (5), 589-600.

\section{Apéndice. Cuestionarios pro- puestos a los estudiantes y breve comentario sobre respuestas ci- entíficamente correctas a los mis- mos}

El número entre paréntesis indica la cantidad de alumnos interrogados en cada caso.

$1-(\mathrm{N}=16)$

a) Dibuje las líneas de campo magnético para un imán como el de la figura.

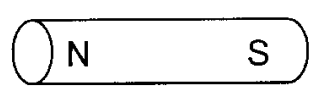

b) Se coloca una placa infinita de madera perpendicular al imán por su punto medio. Dibuje las líneas de campo magnético del imán.

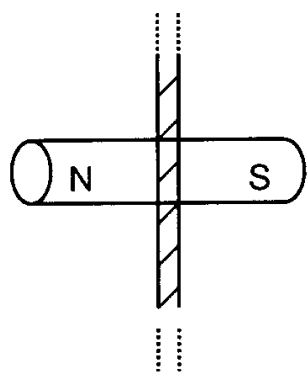

$\mathbf{2}-(\mathbf{N}=\mathbf{1 6})$

a) Dibuje las líneas de campo eléctrico para dos cargas de igual valor absoluto y signos diferentes.

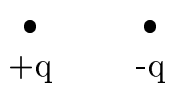

b) Entre esas dos cargas se interpone una placa infinita de madera. Dibuje nuevamente las líneas de campo eléctrico del dipolo. 
<smiles>[TeH][TeH]</smiles>

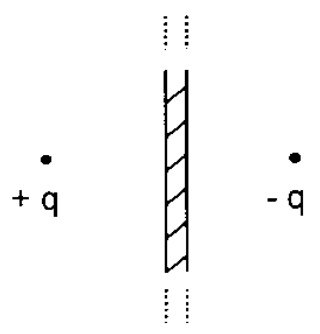

Comentario: Las líneas de campo del imán (en el problema 1) o del dipolo (en el problema 2) no se modifican por la presencia de la placa, ya que el campo creado por un determinado sistema de cargas o imanes es independiente de la presencia de otros objetos. Señalemos que esta afirmación no es válida para el campo resultante, el cual no es el mismo en los apartados a) y b) de aquellos problemas.

\section{$3-(\mathrm{N}=12)$}

La figura muestra una carga positiva Q ubicada a una cierta distancia de una placa infinita de madera. ¿Produce la carga Q campo eléctrico en el punto $\mathrm{P}$, ubicado detrás de la placa?.

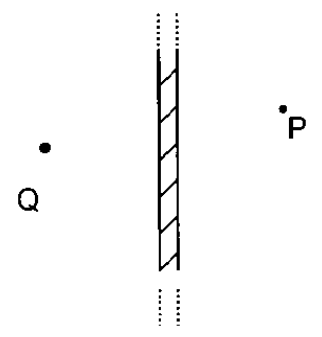

Explique su respuesta.

$4-(\mathrm{N}=6)$

Frente a un imán se coloca una placa infinita de maderą Habrá campo magnético debido a ese imán en el punto $\mathrm{P}$ ubicado detrás de la placa?.

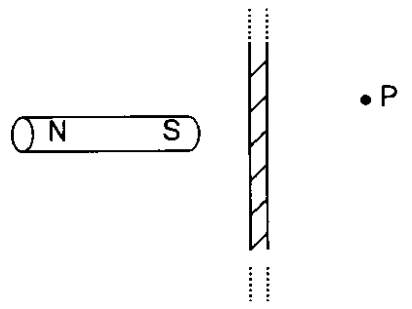

Explique su respuesta.

\section{5- $(\mathrm{N}=15)$}

Una carga Q se encuentra dentro de un hueco practicado en un medio conductor, como se muestra en la figura. ¿Produce la carga Q un campo eléctrico en un punto P del conductor?. Explique su respuesta.

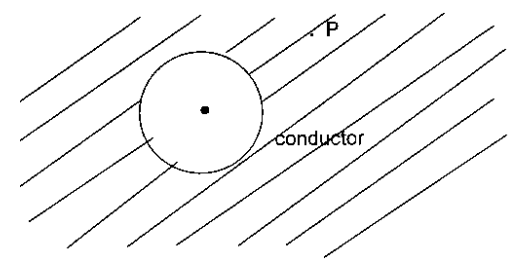

$6-(\mathrm{N}=16)$

Considere un conductor hueco de forma irregular y una carga $\mathrm{Q}$ interior a él. ¿Esa carga $\mathrm{Q}$ crea un campo eléctrico en un punto $\mathrm{P}$ exterior al conductor?.

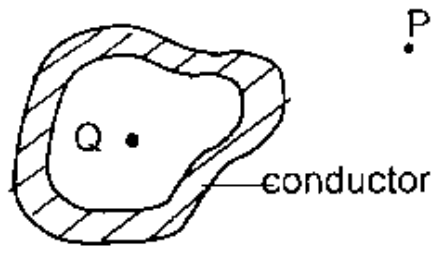

Explique su respuesta.

Comentario: en los problemas $3,4,5$ y 6 la respuesta correcta es "sí". La carga Q (o el imán) produce un campo eléctrico (o magnético) en todos los puntos del espacio, en particular en el punto P. Nótese que el campo de la carga Q (o del imán) en $\mathrm{P}$ no coincide con el campo total en ese punto. Éste resulta de la superposición de las contribuciones generadas por la carga Q y la placa polarizada (en el problema 3), el imán y la placa magnetizada (en el problema 4), la carga Q y las cargas inducidas en la superficie del conductor (en el problema 5, dando en este caso una resultante nula en P) o la carga $Q$ y las cargas inducidas en ambas superficies del conductor hueco (en el problema 6).

$7-(\mathrm{N}=11)$

Dibuje el campo eléctrico creado por una carga $Q$ $=2 \mathrm{C}$ en un punto $\mathrm{P}$, para los tres casos siguientes:
a)
$\dot{\mathrm{Q}}$
$\bullet$
b) $\stackrel{\bullet}{\mathrm{Q}}$
- En P hay una carga $q=-1 \mathrm{C}$
c) $\dot{\mathrm{Q}}$
- En P no hay una carga eléctrica

Comentario: El campo creado por Q en $\mathrm{P}$ es independiente de la presencia de una carga de prueba en ese punto.

\section{$8-(\mathrm{N}=11)$}

En la figura se representa un cascarón conductor cargado positivamente y la superficie de la tierra. El 
cascarón se conecta a tierra mediante un hilo conductor. ¿Habrá circulación de carga?. Explique su respuesta. Si ud. cree que circulará carga, diga cuándo cesa esa circulación.

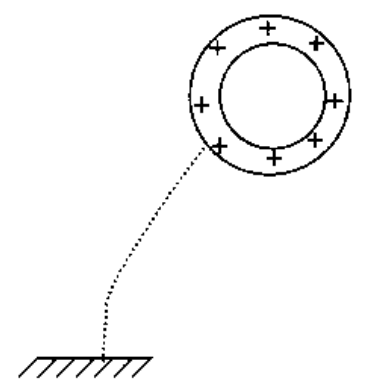

9 - $(\mathrm{N}=18)$

Considere un conductor en cuyas cercanías hay una carga positiva Q. El conductor está conectado a tierra. El sistema está en equilibrio.

a) ¿Es nulo el potencial eléctrico en el conductor?.

b) ¿Es nulo el potencial eléctrico de la tierra?. Explique sus respuestas.

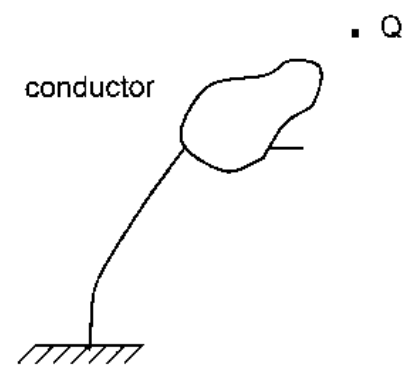

Comentario ( 8 y 9): "la expresión conectada a tierra significa que la superficie u objeto se mantiene al mismo potencial que el punto del infinito por medio de un hilo fino conductor que se intercala entre ellos. Tal conexión se supone que no es capaz de alterar la distribución de potencial. Sin embargo, pueden fluir del infinito al objeto cantidades arbitrarias de carga de uno u otro signo cuya misión es mantener el potencial "a tierra" (usualmente tomado como potencial cero)" (Jackson 1966).

Al conectar a tierra el conductor del problema 8, se producirá un flujo de carga positiva del conductor a la tierra (o un flujo de carga negativa de la tierra al conductor) hasta que el conductor alcance el potencial de la tierra, pues esa es la condición de equilibrio electrostático para el sistema.
Señalemos que en el equilibrio, el conductor del problema 8 quedará descargado. En efecto, si después de la conexión el cuerpo tuviera una carga neta, por su simetría esférica produciría un campo eléctrico radial y habría una diferencia de potencial entre el conductor y la tierra.

En el problema 9, el conductor y la tierra en equilibrio tendrán el mismo potencial eléctrico (que podría elegirse arbitrariamente nulo). Insistimos: esa es la condición de equilibrio electrostático para un sistema de conductores.

Señalemos que en presencia de la carga Q, el conductor del problema 9 adquiere una carga neta negativa. En efecto, la aproximación de la carga $\mathrm{Q}$ eleva el potencial del conductor (con respecto al infinito) por lo que debe fluir carga negativa de la tierra hacia el conductor hasta que éste alcanza nuevamente un potencial igual al de tierra.

\section{$10-(\mathrm{N}=14)$}

En la figura se representan dos esferas conductoras cargadas. Las dos esferas tienen cargas iguales. La esfera A tiene la mitad de radio que la $\mathrm{B}$. Se conectan mediante un hilo conductor. ¿Se producirá una circulación de cargas?. Explique su respuesta. Si ud. cree que circulará carga, diga cuando cesa esa circulación.

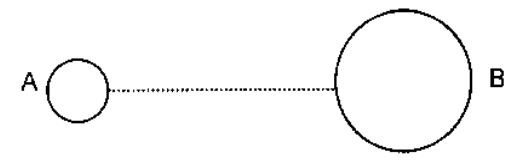

Las dos esferas tienen cargas iguales.

Comentario: suponiendo que el radio de cada esfera sea mucho menor que la distancia entre ellas, puede despreciarse la interacción entre esos cuerpos y considerar uniforme la densidad superficial de carga en cada uno de ellos.

En estas condiciones el potencial eléctrico en cada esfera será inversamente proporcional a cada radio. Por tanto, al tener cargas idénticas, el potencial de la esfera pequeña será mayor que el potencial de la esfera grande. Entonces, al conectar mediante un hilo conductor circulará carga desde el cuerpo pequeño hacia el grande hasta que el potencial eléctrico sea el mismo en todos los puntos del sistema, pues ésta es la condición de equilibrio electrostático para un conductor cargado.

\section{$11-(\mathrm{N}=13)$}

En la situación representada en la figura, las dos superficies equipotenciales son planos infinitos, perpendiculares al plano de la figura, y el espacio entre esas superficies está ocupado por distintos materiales. En la zona I (de la izquierda) hay un dieléctrico de constante $k_{1}$, en la zona II (la del centro) hay un material conductor, y en la zona III (de la derecha) hay otro dieléctrico de constante $k_{2}\left(k_{1}<k_{2}\right)$. 


\section{superficie equipotencial (potencial $V_{1}$ )}

\section{superficie equipotencial (potencial $V_{1}$ )}

Marque las opciones que comparta, referida al campo eléctrico entre las placas y explique su respuesta. El campo eléctrico:

..... es el mismo en las tres zonas

..... tiene diferente módulo en cada zona (explique)

..... es nulo en la zona II (material conductor)

..... no sé

Comentario: El campo eléctrico es el mismo en las tres regiones, pues la diferencia de potencial es idéntica en esas tres zonas.

La simetría del problema indica que el campo eléctrico será uniforme. Éste puede calcularse como el producto de la diferencia de potencial y la distancia entre las placas equipotenciales infinitas.

$12-(\mathrm{N}=14)$

Una carga $\mathrm{Q}=+2 \mathrm{C}$ se encuentra a una cierta distancia de un punto P. Considere las tres situaciones diferentes:
a) $\stackrel{\bullet}{\bullet} \quad \stackrel{P}{\mathrm{P}}$
En $\mathrm{P}$ hay una carga $\mathrm{q}=+1 \mathrm{C}$
b) $\stackrel{\bullet}{\mathrm{Q}} \quad \stackrel{\mathrm{P}}{\mathrm{P}}$
En $P$ hay una carga $q=-1 C$
c)

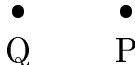

Marque con una cruz los enunciados correctos y explique su respuesta.

El potencial eléctrico en el punto $P$ :

..... es el mismo en los tres casos

..... tiene signos distintos en a) y b)

..... es nulo en c)

..... otra opción (explique)

..... no sé

Comentario: la opción correcta es la que afirma que el potencial eléctrico es el mismo en los tres casos, ya que éste depende de la carga $Q$, fuente de dicho potencial y no depende de la magnitud, el signo o la presencia de una carga de prueba en el punto $\mathrm{P}$.

$13-(\mathrm{N}=16)$

Considere dos cargas puntuales positivas, Q y Q', situadas en dos puntos $\mathrm{P}_{1}$ y $\mathrm{P}_{2}$, respectivamente. Sea $Q^{\prime}=2 \mathrm{Q}$

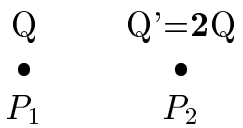

Indique la opción correcta y explique su respuesta. El potencial eléctrico en $P_{1}$ es

mayor ..... menor ...... igual ...... no sé ...... que el potencial eléctrico en $P_{2}$.

Comentario: la opción correcta afirma que: "el potencial eléctrico en $P_{1}$ es mayor que el potencial eléctrico en $P_{2}$ ", pues en $P_{1}$ la fuente de potencial es la carga más grande, Q', mientras que en $P_{2}$ la fuente es $\mathrm{Q}$.

\section{$14-(\mathrm{N}=12)$}

Para la siguiente afirmación elija una de las opciones indicadas y explique su respuesta: "una carga positiva produce un potencial eléctrico positivo en todos los puntos del espacio que la rodea".

correcto ...... incorrecto ...... no sé ......

Comentario: El valor del potencial eléctrico producido por un cuerpo cargado en un punto se define con respecto a una cierta posición arbitraria de referencia. Es decir, el potencial en un punto es la diferencia de potencial entre ese punto y el que se adopta como referencial.

Por lo tanto, la magnitud y el signo de $\mathrm{V}$ en cada punto del espacio no dependerán solamente de la carga fuente, sino también del referencial elegido.

La afirmación del problema puede considerarse correcta si se ha elegido el referencial a una distancia infinita de la carga fuente, pero es incorrecta si se eligió otra posición como referencia para el potencial. 


\section{$15-(\mathrm{N}=16)$}

Para el siguiente enunciado, diga si lo considera verdadero o falso y explique su respuesta.

"Una carga puntual positiva sólo puede tener energía potencial eléctrica positiva".

verdadero ...... falso ...... no sé ......

Comentario: la energía potencial eléctrica de una carga puntual, $U_{\text {pot }}=q . V_{\text {ext }}$, depende de la carga y del potencial eléctrico externo en la posición de aquélla. A su vez, dicho potencial estará determinado por la distribución fuente que lo genera y por el referencial adoptado para especificarlo.

Por lo tanto, la afirmación enunciada en el problema es falsa. El signo de la energía potencial de una carga puntual positiva puede ser positivo o negativo, dependiendo de la distribución de carga que produce el potencial exterior y de la posición del punto donde éste potencial se considere arbitrariamente nulo.

$16-(\mathrm{N}=20)$

$\mathrm{Al}$ calcular la energía potencial eléctrica de una carga Q ubicada en la región comprendida entre las placas de un capacitor, un alumno A afirma que todos los estudiantes deben obtener el mismo valor numérico. Otro alumno B sostiene que diferentes estudiantes pueden obtener valores numéricos diferentes.

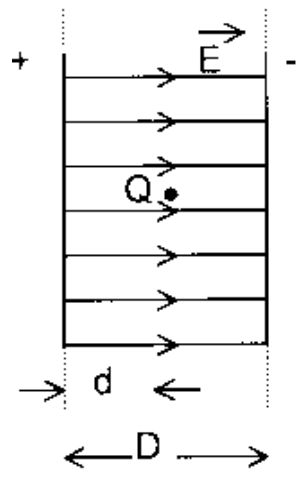

Marque la opción que Ud. comparte y explique su respuesta:

A tiene razón...... B tiene razón ...... no sé .......

Comentario: diferentes estudiantes pueden obtener valores numéricos distintos si ellos adoptan distintos referenciales para especificar la energía potencial eléctrica de la carga $Q$.

Por lo tanto, la opción correcta es la que afirma que "B tiene razón". 